\title{
Ab initio Defect Energetics in LaBO3 Perovskite Solid Oxide Fuel Cell Materials
}

\author{
Lee, Yueh-Lin; Morgan, Dane; Kleis, Jesper; Rossmeisl, Jan
}

Published in:

E C S Transactions

Link to article, DOI:

$10.1149 / 1.3205837$

Publication date:

2009

Document Version

Publisher's PDF, also known as Version of record

Link back to DTU Orbit

Citation (APA):

Lee, Y-L., Morgan, D., Kleis, J., \& Rossmeisl, J. (2009). Ab initio Defect Energetics in LaBO3 Perovskite Solid Oxide Fuel Cell Materials. E C S Transactions, 25(2), 2761-2767. https://doi.org/10.1149/1.3205837

\section{General rights}

Copyright and moral rights for the publications made accessible in the public portal are retained by the authors and/or other copyright owners and it is a condition of accessing publications that users recognise and abide by the legal requirements associated with these rights.

- Users may download and print one copy of any publication from the public portal for the purpose of private study or research.

- You may not further distribute the material or use it for any profit-making activity or commercial gain

- You may freely distribute the URL identifying the publication in the public portal

If you believe that this document breaches copyright please contact us providing details, and we will remove access to the work immediately and investigate your claim. 


\title{
$A b$ initio Defect Energetics in $\mathrm{LaBO}_{3}$ Perovskite Solid Oxide Fuel Cell Materials
}

\author{
Y.-L. Lee ${ }^{\mathrm{a}}$, D. Morgan ${ }^{\mathrm{a}, \mathrm{b}}$, J. Kleis $^{\mathrm{c}}$, J. Rossmeisl $^{\mathrm{c}}$ \\ ${ }^{a}$ Materials Science Program, University of Wisconsin-Madison, Madison \\ Wisconsin 53706, USA \\ ${ }^{\mathrm{b}}$ Department of Materials Science and Engineering, University of Wisconsin-Madison \\ Madison, Wisconsin 53706, USA \\ ${ }^{\mathrm{c}}$ Center for Atomic-scale Materials Design (CAMD), Department of Physics \\ Building 307, Technical University of Denmark, Denmark
}

\begin{abstract}
Perovskite materials of the form $\mathrm{ABO}_{3}$ are a promising family of compounds for use in solid oxide fuel cell (SOFC) cathodes. Study of the physics of these compounds under SOFC conditions with $a b$ initio methods is particularly challenging due to high temperatures, exchange of oxygen with $\mathrm{O}_{2}$ gas, and correlated electron effects. This paper discusses an approach to performing ab initio studies on these materials for SOFC applications and applies the approach to calculate oxygen vacancy formation energies in $\mathrm{LaBO}_{3}(\mathrm{~B}=\mathrm{Mn}$, $\mathrm{Fe}, \mathrm{Co}, \mathrm{Ni}$ ) compounds.
\end{abstract}

\section{Introduction}

Solid oxide fuel cells cathodes must catalyze the oxygen reduction reaction (ORR), which consists of the reaction $\mathrm{O}_{2}$ (gas) $\rightarrow 2 \mathrm{O}^{2-}$ (bulk). The oxygen is initially in the gas phase, and after reduction must be incorporated into bulk for transport through the electrolyte to the anode. Perovskite oxides (with formula unit $\mathrm{ABO}_{3}$, where $\mathrm{A}$ and $\mathrm{B}$ are cations or sets of cations) are active for the ORR under SOFC conditions (generally 700 $K-1200 \mathrm{~K}$ and air atmosphere) and have a number of advantages over competing materials, including their stability at high temperature, reasonable cost, and acceptable thermal expansion properties $(1,2)$. Nonetheless, SOFC efficiency is significantly reduced by overpotential in the cathodic ORR, a problem that is likely to get worse as researchers push to lower temperatures and thinner electrolyte SOFCs (2). Therefore, there is increasing interest in enhancing the catalytic ability of perovskite cathodes. In order to better understand the ORR on perovskite materials it is necessary to understand the defect structure of the perovskite, which can strongly influence such processes as oxygen dissociation on the cathode surface, incorporation into the cathode bulk, and transport (either by surface or bulk route) to the electrolyte (1).

$A b$ initio density functional theory (DFT) methods are powerful tools for modeling oxide properties (3) and defect chemistry. However, the energetics at SOFC cathodes are particular challenging to model, as careful treatment of the correlated electron effects introduced by the B site transition metal cations (in our case, Mn, Co, Fe and Ni), JahnTeller distortions, and magnetic ordering is necessary. Also the oxygen gas reference state must be corrected to properly describe the oxygen exchange reactions underlying the ORR energetics. Finally, the defect physics predicted by zero-temperature $(T=0 \mathrm{~K})$ 
DFT approaches must be supplemented with proper thermodynamics to yield values accurate at SOFC operating temperatures.

This paper discusses the issues of adapting density functional theory methods to study the defects in perovskite SOFC cathodes. We focus on oxygen vacancy energetics $\mathrm{LaBO}_{3}$ ( $\mathrm{B}=\mathrm{Mn}, \mathrm{Fe}, \mathrm{Co}, \mathrm{Ni}$ ) compounds, which are similar to commonly used perovskite cathodes such as $(\mathrm{La}, \mathrm{Sr}) \mathrm{MnO}_{3}$ and $(\mathrm{La}, \mathrm{Sr})(\mathrm{Co}, \mathrm{Fe}) \mathrm{O}_{3}(1)$.

\section{General Computational Methods}

The results presented here are based on spin polarized DFT calculations, performed with the Vienna Ab-initio Simulation Package (VASP) (4) using a plane wave basis set, the GGA-Perdew-Wang-91 (5) exchange correlation function, and the Projector Augmented Wave (PAW) method $(6,7)$. We used PAW potentials with names and electronic configurations La (5s2 5p6 6s2 5d1), Os (2s2 2p4), B = Mn_pv (3p4s3d), Fe_pv (3p6 3d7 4s1), Co (3d8 4s1), and Ni_pv (3d9 4s1). All calculations were done with an energy cut-off of $600 \mathrm{eV}$. A $2 \times 2 \times 2$ Monkhorst-Pack $k$-point mesh in the Brillouin zone was used for a $2 \times 2 \times 2$ supercell (containing 40 total atoms) of the primitive perovskite formula unit. The energies are converged to within $\sim 3 \mathrm{meV}$ per atom with respect to the $k$-points and energy cutoff. Structural relaxations are converged to within $1 \mathrm{meV}$ per atom. The rotationally invariant $\mathrm{GGA}+\mathrm{U}$ approach (8) in the simplified spherically averaged version (9) was used to treat correlation effects. In this approach the Hubbard Hamiltonian can be represented by a single $U_{\text {eff }}=U-J$. We take $J=1 \mathrm{eV}$ in all cases and $U_{\text {eff }}=4.0 \mathrm{eV}, 4.0 \mathrm{eV}, 3.3 \mathrm{eV}$, and $6.4 \mathrm{eV}$ for $\mathrm{Mn}, \mathrm{Fe}, \mathrm{Co}$, and $\mathrm{Ni}$, respectively. These values were optimized by Wang, et al to give accurate oxidation/reduction energetics (10).

\section{Magnetism and Jahn-Teller Distortions}

The perovskites can have quite complex magnetic orderings that depend sensitively on temperature, alloying, and oxygen stoichiometry (or equivalently, oxygen partial pressure) (11). At the high temperatures appropriate for SOFC conditions, magnetic ordering will generally be paramagnetic. In order to avoid the complexity of accurately modeling the magnetic state, all calculations presented in this work are done with ferromagnetic ordering. This is an approximation both for the energetics and the electronic structure, as antiferromagnetic ordering can alter the band structure and metallic character. However, energy differences between different magnetic orderings are typically in the range of 0.01-0.1 eV/magnetic ion, which is significantly smaller than most defect energies (e.g., vacancy formation energies are typically a few $\mathrm{eV}$ ). Therefore, it is likely that this approximate treatment of magnetism will introduce only small percentage errors in the ab initio defect energetics.

Similarly to magnetic structure, the Jahn-Teller distortions in the perovskites are sensitive to temperature and stoichiometry, and are challenging to model. In particular, stoichiometric $\mathrm{LaMnO}_{3}$ and $\mathrm{LaCoO}_{3}$ have significant Jahn-Teller distortions at lower temperatures, but become cubic at $\mathrm{T}_{\mathrm{JT}}=750 \mathrm{~K}$ and $\mathrm{T}_{\mathrm{JT}}=500 \mathrm{~K}$, respectively $(12,13)$. However, even in the high-temperature cubic phase it is likely that there is at least some local distortion associated with the Jahn-Teller effect. In order to include both the average 
cubic nature of the high-temperature phase and the effects of local distortions, lattice parameters are initially calculated from full relaxation of an ideal cubic perovskite unit cell, and then all bulk calculations are done with internal relaxation of $2 \times 2 \times 2$ supercells with the obtained lattice parameters. This partially constrained geometry is used in the $T$ $=0 \mathrm{~K}$ calculations to approximately mimic the structures, and therefore the energetics, that are likely at SOFC operating temperatures. The partially constrained geometry approach for a $2 \times 2 \times 2$ cell completely suppresses the Jahn-Teller distortions in the undefected bulk, which is likely to create an artificially high destabilization of the bulk enthalpy. However, the relaxations do allow for local distortions associated with the Jahn-Teller effect to occur when defects are introduced, e.g., by a vacancy. The JahnTeller distortions are typically less than $0.3 \mathrm{eV} / \mathrm{Jahn}-\mathrm{Teller}$ ion (14). Specifically, our calculation results suggest the fully relaxed $2 \times 2 \times 2 \mathrm{LaMnO}_{3}$ and $\mathrm{LaCoO}_{3}$ perovskites are about $0.05 \mathrm{eV} / \mathrm{Jahn}$-Teller ion more stable than the constrained $2 \times 2 \times 2$ cubic supercells. Therefore, as with the magnetic approximations, we expect this approximate treatment of Jahn-Teller effects will introduce only small percentage errors in the ab initio defect energetics.

\section{DFT Corrections for $\mathrm{O}_{2}$ Gas and Correlation Effects}

In this section we investigate the bulk oxygen vacancy formation energy at $T=0 \mathrm{~K}$ and compare our results with experimentally obtained vacancy formation enthalpy values.
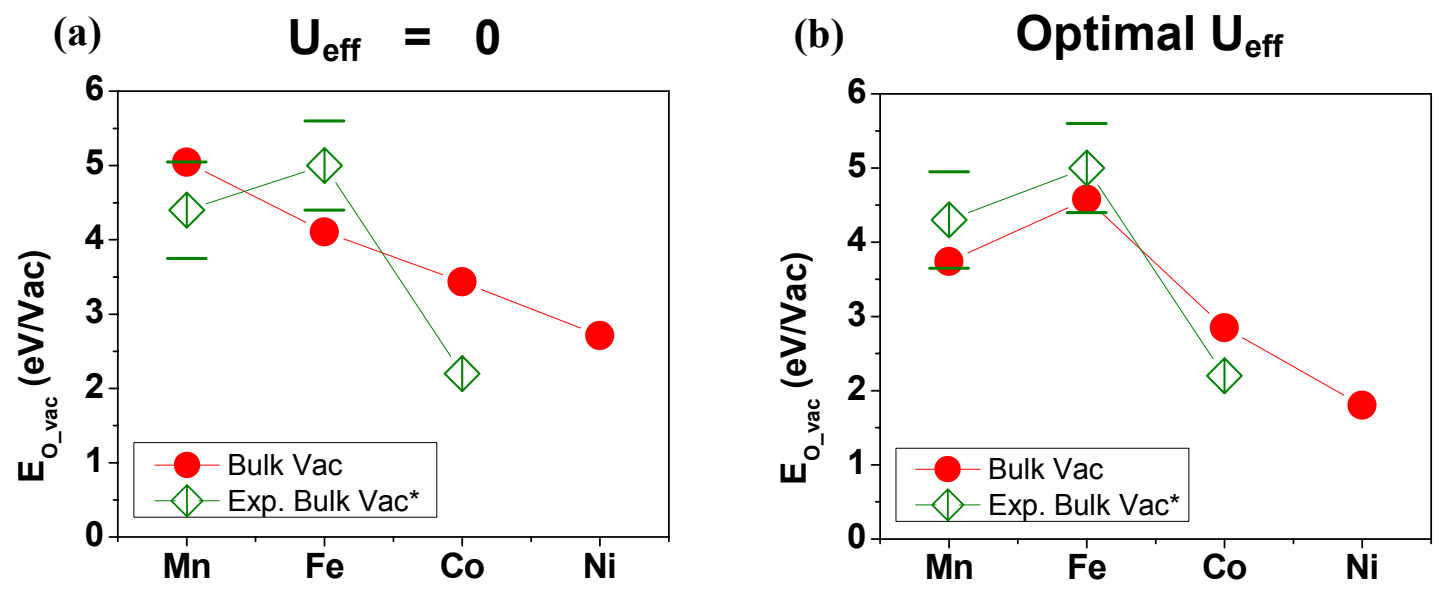

Figure 1. Oxygen vacancy formation enthalpies for stoichiometric bulk $\mathrm{LaBO}_{3}(\mathrm{~B}=\mathrm{Mn}$, $\mathrm{Fe}, \mathrm{Co}, \mathrm{Ni}$ ) calculated based on ab initio energies and from experimental results. Results are given at $T=0 \mathrm{~K}$ from the calculations for $\mathrm{U}_{\mathrm{eff}}=0$ and optimal $\mathrm{U}_{\text {eff }}$ from Ref. (10) ( $U_{\text {eff }}=4.0 \mathrm{eV}, 4.0 \mathrm{eV}, 4.3 \mathrm{eV}$, and $6.4 \mathrm{eV}$ for $\mathrm{Mn}, \mathrm{Fe}, \mathrm{Co}$, and $\mathrm{Ni}$, respectively). The experimental oxygen vacancy formation enthalpy data (with error bars) are taken from $(15,16)$ for $\mathrm{LaMnO}_{3}$, (17) for $\mathrm{LaFeO}_{3}$, and (18) for $\mathrm{LaCoO}_{3}$.

The $T=0 \mathrm{~K}$ calculated bulk vacancy formation energy is very close in value to the vacancy formation enthalpy, and is given by

$$
\Delta E_{V f}=E_{\mathrm{LaBO}_{3}}^{a b i n i t}(v a c)-E_{\mathrm{LaBO}_{3}}^{a b i n i t}+\frac{1}{2}\left(E_{\mathrm{O}_{2}}^{a b i n i t}+\Delta h_{\mathrm{O}_{2}}^{0}\right)
$$


where $E_{\mathrm{LaBO}_{3}}^{a b i n i t}(v a c)$ and $E_{\mathrm{LaBO}_{3}}^{a b i n i t}$ are the energies of the stoichoimetric compound with and without an oxygen vacancy, respectively. $E_{O_{2}}^{\text {abinit }}$ is the ab initio calculated energy of the $\mathrm{O}_{2}$ molecule and $\Delta h_{\mathrm{O}_{2}}^{0}$ is a correction term, which accounts for errors that do not cancel between the treatment of oxygen in the gas and solid phases. Following the approach of Wang, et al. (10), we calculate $\Delta h_{\mathrm{O}_{2}}^{0}=0.33 \mathrm{eV} / \mathrm{O}_{2}$ for our choices of exchange correlation and pseudopotentials.

Besides the molecular oxygen correction, the correlated electron effects introduced by the B site transition metal have to be properly treated. We use the GGA+U method, and Figure 1 demonstrates that this method consistently improves the oxygen vacancy formation enthalpy compared to standard GGA $\left(U_{\text {eff }}=0\right)$.

\section{Oxygen Vacancy Formation Energetics at Elevated Temperatures}

Many properties relevant for the ORR (e.g., oxygen vacancies and adsorbed oxygen on the surface) include the movement of oxygen from the solid perovskite to oxygen gas. In order to correctly model the free energies of reaction for oxygen with the solid at elevated temperatures, it is necessary to have the correct chemical potential of gas phase oxygen, with the same reference as the ab initio bulk oxygen. The chemical potential of oxygen can be written $(10,19,20)$ as,

$$
\mu_{O}\left(T, P, O_{\text {abinit }}^{r e f}\right)=\frac{1}{2}\left(\begin{array}{l}
E_{O_{2}}^{a b i n i t}+\Delta h_{O_{2}}^{0} \\
+\left(H_{O_{2}}\left(T, P^{0}\right)-H_{O_{2}}\left(T^{0}, P^{0}\right)\right)-T S_{O_{2}}\left(T, P^{0}\right) \\
+k T \ln \left(\frac{P}{P^{0}}\right)
\end{array}\right)
$$

where $\mu_{O}\left(T, P, O_{a b i n i t}^{r e f}\right)$ is the chemical potential for oxygen at temperature $T$ and partial pressure $P$, referenced to the energy of oxygen in the ab initio calculations. The molecular oxygen term with corrections was introduced in the previous section. The terms on the second line are the free energy of oxygen gas at $T, P$ relative to the enthalpy at standard conditions, which we obtain from Ref. (21). The final term is the usual expression for change in chemical potential with pressure for a gas. The oxygen vacancy formation free energy (which is the free energy to form an oxygen vacancy without any contribution from the bulk configurational degrees of freedom) can now be obtained from

$$
\begin{aligned}
& \Delta G_{V f}^{*}=G_{\mathrm{LaBO}_{3}}(v a c)-G_{\mathrm{LaBO}_{3}}+\mu_{O} \\
& =E_{\mathrm{LaBO}_{3}}^{a b i n i t}(v a c)-E_{\mathrm{LaBO}_{3}}^{a b i n i t}+\underbrace{\left(\mu_{O}-\frac{1}{2}\left(\bar{G}_{O_{2}}^{s, v i b}(T)-\bar{H}_{O_{2}}^{s, v i b}\left(T^{0}\right)\right)\right)}_{\mu_{O}^{e f f}}
\end{aligned}
$$

where we separate out temperature dependent behavior, including solid phase vibrational terms and the oxygen chemical potential, into an effective parameter $\mu_{O}^{\text {eff }}$. $\mu_{O}^{\text {eff }}$ includes 
the vibrational effects in the solid $\frac{1}{2}\left(\bar{G}_{O_{2}}^{s, v i b}(T)-\bar{H}_{O_{2}}^{s, v i b}\left(T^{0}\right)\right)$, where $\bar{G}_{O_{2}}^{s, v i b}(T)$, and $\bar{H}_{\mathrm{O}_{2}}^{s, v i b}\left(T^{0}\right)$ are the vibrational contribution to the partial molar Gibbs energy and enthalpy of $\mathrm{O}_{2}$ in the solid, respectively. The enthalpy at standard temperature $\left(T^{0}=298.15 \mathrm{~K}\right)$ has to be removed from $\bar{G}_{O_{2}}^{s, v i b}(T)$ as it is already effectively included in $\Delta h_{O_{2}}^{0}$ due to the way it is fit. The partial molar vibrational terms are obtained from a simple Einstein model for the vibrations of the oxygen, where an Einstein temperature of $\theta_{E}=500 \mathrm{~K}$ is used. This temperature is obtained from $a b$ initio calculation of force constants in a local harmonic model (22) of the vibrating oxygen in $\mathrm{LaMnO}_{3}$. While this treatment of the solid phase oxygen thermodynamics is quite approximate, it is clearly better than ignoring the vibrational contributions to the solid phase from the oxygen altogether, particularly as the vibrational contribution from that same oxygen do enter into the gas chemical potential $\left(\mu_{O}\right)$ implicitly through the experimental data. The overall effect of including the solid phase oxygen vibrations on the vacancy formation free energy is about $3 k T$ per oxygen for $T>\theta_{E}$, or approximately $0.25 \mathrm{eV} /$ oxygen at $1000 \mathrm{~K}$. Therefore, while this effect is significant, even an error of $50 \%$ will not make a large impact on vacancy formation energies of a few $\mathrm{eV}$.

Figure 2 is a plot of the function $\mu_{O}^{e f f}-\frac{1}{2}\left(E_{O_{2}}^{\text {vasp }}+\Delta h_{O_{2}}^{0}\right)$ as a function of temperature at $P\left(\mathrm{O}_{2}\right)=0.2 \mathrm{~atm}$. The term $\mu_{O}^{\text {eff }}-\frac{1}{2}\left(E_{\mathrm{O}_{2}}^{\text {vasp }}+\Delta h_{O_{2}}^{0}\right)$ would be added to the vacancy formation enthalpy at $T=0 K$ (see Equation 1) to obtain the vacancy formation free energy at finite $T>0 \mathrm{~K}$ (see Equation 3). The values in Figure 2 range from about $-0.3 \mathrm{eV}$ at $300 \mathrm{~K}$ to about $-1.2 \mathrm{eV}$ at $1273 \mathrm{~K}$, which is near the upper range for SOFC operation. This energy will lower the vacancy formation free energy, making it easier to form vacancies at higher temperature. This effect is primarily due to the large entropy of the $\mathrm{O}_{2}$ gas, which stabilizes the oxygen leaving the solid phase. However, the vibrational energetics of the solid phase oxygen makes a significant contribution $(\sim 20 \%)$ to the energy in Figure 2 at $1000 \mathrm{~K}$. It is therefore important that all the thermodynamic terms giving rise to the values in Figure 2 be included at higher temperatures.

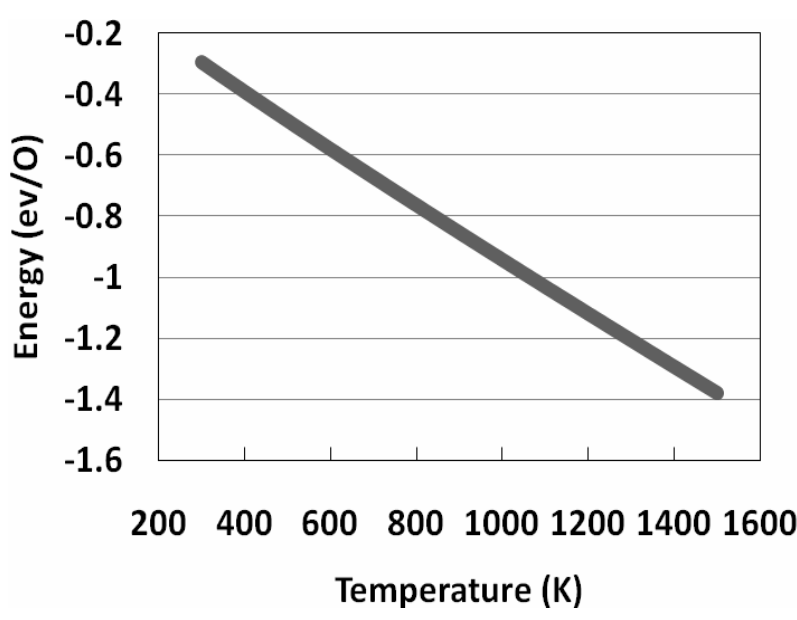

Figure 2. The calculated $\mu_{O}^{\text {eff }}-\frac{1}{2}\left(E_{O_{2}}^{\text {vasp }}+\Delta h_{O_{2}}^{0}\right)$ vs. $T$ at $P\left(\mathrm{O}_{2}\right)=0.2 \mathrm{~atm}$. This term would be added to the $T=0 \mathrm{~K}$ vacancy formation enthalpy to correct for temperature effects. 
Although the stabilization effects of temperature in Figure 2 can be as large as $-1.2 \mathrm{eV}$, the oxygen vacancy formation free energies for all the systems studied are still positive. In fact, in all cases except $\mathrm{Ni}$ the defect formation free energies even at $1273 \mathrm{~K}$ are well over $1 \mathrm{eV}$, which is one of the reasons doping with $\mathrm{Sr}$ or other $2+$ cations on the A site is typically used to enhance vacancy concentrations.

\section{Summary and Conclusions}

In summary, we have developed an approach to calculate energetics of perovskite oxides relevant for SOFC cathodes and the oxygen reduction reaction. The approach is practical to implement on these complex systems and includes at least an approximate treatment of magnetism, Jahn-Teller effects, the thermodynamics of interaction with $\mathrm{O}_{2}$ gas at high temperature, and correlated electron properties. We have used the approach to calculate bulk oxygen vacancy formation enthalpies and formation free energies for $\mathrm{LaBO}_{3}(\mathrm{~B}=\mathrm{Mn}, \mathrm{Fe}, \mathrm{Co}, \mathrm{Ni})$ compounds. The results are in good agreement with experimental values and demonstrate the importance of correctly treating the thermodynamic effects at high temperatures and errors associated with electron correlation.

\section{Acknowledgments}

We gratefully acknowledge financial support from NSF MRSEC program (0079983)

and computing support from NSF National Center for Supercomputing Applications (NCSA - DMR060007).

\section{References}

1. S. B. Adler, Chemical Reviews, 104, 4791 (2004).

2. S. C. Singhal and K. Kendall, High Temperature Solid Oxide Fuel Cells: Fundamentals, Design and Applications, p. 512, Elsevier Science (2004).

3. G. Ceder, A. Van der Ven, C. Marianetti and D. Morgan, Modelling and Simulation in Materials Science and Engineering, 8, 311 (2000).

4. G. Kresse and J. Furthmuller, Physical Review B, 54, 11169 (1996).

5. J. P. Perdew, K. Burke and M. Ernzerhof, Physical Review Letters, 77, 3865 (1996).

6. P. E. Blochl, Physical Review B, 50, 17953 (1994).

7. G. Kresse and D. Joubert, Physical Review B, 59, 1758 (1999).

8. A. I. Liechtenstein, V. I. Anisimov and J. Zaanen, Physical Review B, 52, R5467 (1995).

9. S. L. Dudarev, G. A. Botton, S. Y. Savrasov, C. J. Humphreys and A. P. Sutton, Physical Review B, 57, 1505 (1998).

10. L. Wang, T. Maxisch and G. Ceder, Physical Review B, 73 (2006). 
11. L. Ghivelder, I. A. Castillo, M. A. Gusmao, J. A. Alonso and L. F. Cohen, Physical Review B, 60, 12184 (1999).

12. J. S. Zhou and J. B. Goodenough, Physical Review B, 60, R15002 (1999).

13. C. Zobel, M. Kriener, D. Bruns, J. Baier, M. Gruninger, T. Lorenz, P. Reutler and A. Revcolevschi, Physical Review B, 66 (2002).

14. C. A. Marianetti, D. Morgan and G. Ceder, Physical Review B, 63, 224304 (2001).

15. J. H. Kuo, H. U. Anderson and D. M. Sparlin, Journal of Solid State Chemistry, 83, 52 (1989).

16. J. Nowotny and M. Rekas, Journal of the American Ceramic Society, 81, 67 (1998).

17. J. Mizusaki, M. Yoshihiro, S. Yamauchi and K. Fueki, Journal of Solid State Chemistry, 58, 257 (1985).

18. J. Mizusaki, Y. Mima, S. Yamauchi, K. Fueki and H. Tagawa, Journal of Solid State Chemistry, 80, 102 (1989).

19. E. Heifets, J. Ho and B. Merinov, Physical Review B, 75 (2007).

20. Y.-L. Lee, D. Morgan, J. Kleis and J. Rossmeisl, Ab initio Oxygen Reduction Reaction Energetics of LaBO3 (B=Mn, Fe, Co, and Ni) (001) Surfaces for Solid Oxide Fuel Cell Cathodes, Submitted (2009).

21. NIST Chemistry WebBook, NIST Standard Reference Database No. 69, edited by P. J. Linstrom and W. G. Mallard (National Institute of Standards and Technology, Gaithersburg, MD, 2003), http://webbook.nist.gov/chemistry/.

22. L. Zhao, R. Najafabadi and D. J. Srolovitz, Modelling and Simulation in Materials Science and Engineering, 1, 539 (1993). 